\title{
Computation of the Stress Intensity Factor and COD for Submicron Sized Indentation Cracks
}

\author{
Gerold A. SCHNEIDER* and Theo FETT**,***
}

*Institut für keramische Hochleistungswerkstoffe, Technische Universität Hamburg-Harburg, Denickestrasse 15, 21073 Hamburg, Germany

**Institut für Materialforschung II, Forschungszentrum Karlsruhe, Karlsruhe, Germany

***Institut für Keramik im Maschinenbau, Universität Karlsruhe, Karlsruhe, Germany

\begin{abstract}
The first part of the paper deals with the computation of stress intensity factors for pyramidal indentation cracks with a ratio of crack length $c$ to indentation half-diagonal $a$ between 1 and 2 . The residual stress field due to quasi-plastic deformation below the indenter is described by using the Hill model of an expanding sphere. It is found that the proportionality factor in the relation for $K_{\text {Ic }}$ decreases with $c / a \rightarrow 1$ and confirms experimental results with nanoindents on sapphire and fused quartz. In the second part, the crack opening displacement of such cracks is determined.

[Received July 14, 2006; Accepted September 21, 2006]
\end{abstract}

Key-words : Indentation crack length, ICL, Fracture toughness, COD, Stress intensity factor, Nanoindentation

1. Introduction

Indentation tests with pyramidal indenter are very often used for the determination of fracture toughness of brittle materials. Besides its simplicity the indentation test offers the possibility to measure the fracture toughness inside $\mu \mathrm{m}$-sized ceramic grains and nano-sized thin films using nanoindenters.

In the case of glasses and other low-toughness materials, fracture toughness is obtained from the relation

$$
K_{\mathrm{Ic}}=\kappa\left(\frac{E}{H}\right)^{1 / 2} \frac{P}{c^{3 / 2}}
$$

as was proposed by Lawn et al. ${ }^{1)}$ on the basis of half pennyshaped indentation cracks. In Eq. (1) $E$ is Young's modulus, $H$ the hardness, $P$ the indentation load, and $c$ the radius of an assumed semi-circular crack. In the case of a Vickers pyramid with its relatively flat opening angle, the empirical constant $\kappa$ was determined by Anstis et al. ${ }^{2)}$ as $\kappa=0.016$. Applicability of relation (1) was verified by Harding et al. ${ }^{3)}$ for a sharper corner-cube diamond pyramid in a nano-indenter loading system. The related empirical constant $\kappa$ was found to be $\kappa=$ 0.036 .

For tougher materials as e.g. $\mathrm{Si}_{3} \mathrm{~N}_{4}$ or $\mathrm{ZrO}_{2}$-ceramics so called Palmqvist cracks, starting from the pyramid corners and extending very close to the surface are observed. If the crack length at the surface is denoted as $l=c-a$ ( $a=$ halfdiagonal of the pyramid impression), the fracture toughness is given by

$$
K_{\mathrm{Ic}} \propto\left(\frac{E}{H}\right)^{n} \frac{P}{\sqrt{l}}
$$

with the exponent $n$ mostly reported in literature to be $n=0.4$ (e.g. Niihara et al. $^{4)}$ ). Materials which exhibit Palmqvist cracks at lower loads and $c / a<2.5$ show a change in crack type to semi-circular penny shaped cracks at higher loads and $c / a>2.5$. By plotting the crack length $c$ versus $P^{2 / 3}$, it can be decided whether a material behaves according to Eq. (1).

Measurements on fused quartz, sapphire, and barium titanate were carried out by Scholz et al. ${ }^{5)}$ using a corner-cube indenter mounted in a commercial nano-indenter system. The applied loads were between 0.5 and $10 \mathrm{mN}$, resulting in submicron cracks. The crack lengths were determined using AFM line profiles perpendicular to the crack.

Figure 1 (a) shows the plot $c=f\left(P^{2 / 3}\right)$. All materials exhibit a linear dependency that ensures the occurrence of half penny-
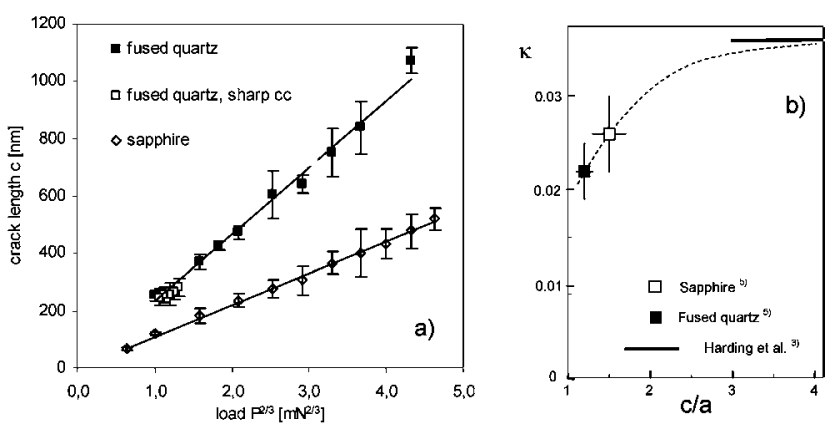

Fig. 1. (a) Crack length in nano-indentation tests with a cornercube indenter for different materials, reported by Scholz et al., ${ }^{5)}$ (b) empirical constant $\kappa$ for different materials.

shaped cracks, although $c / a$ was significantly smaller than 2.5 in two cases (namely, 1.5 for sapphire and 1.2 for fused quartz). Scholz et al. ${ }^{5)}$ found astonishingly small values of the empirical constant $\kappa$, namely, $\kappa=0.026$ for sapphire and 0.022 for quartz, contrary to the value of $\kappa=0.036$ obtained by Harding and co-workers ${ }^{3}$ ) for cracks with $c / a>3$ (Fig. 1 (b)). In order to check whether a change of the empirical constant is a general effect for small $c / a$ ratios independent of the special indenter type, computations will be performed for the case of half penny-shaped cracks as resulting for instance in Vickers indentation tests.

For this purpose, the stress intensity factor will be determined on the basis of the residual stress field given by Hill. ${ }^{6}$ ) This model providing the correct $c \propto P^{2 / 3}$ dependency for $c / a$ $>3$ as outlined in $^{7)}$ shall be applied to cracks with a smaller $c / a$ ratio.

2. Computation of the stress intensity factor and COD

Beneath the contact area of a Vickers indenter pressed into the surface of a brittle material, a residual stress zone remains after unloading. The pressure distribution in this region may be described as proposed by Hill ${ }^{6)}$

$$
\sigma_{\text {res }}(r)=\left\{\begin{array}{cll}
-k \sigma_{0} & \text { for } \quad r<c_{1} \\
\sigma_{0}\left((k+1) \frac{r-c_{1}}{c_{2}-c_{1}}-1\right) & \text { for } c_{1}<r<c_{2} \\
\sigma_{0}\left(c_{2} / r\right)^{3} & \text { for } \quad c_{2}<r
\end{array}\right.
$$



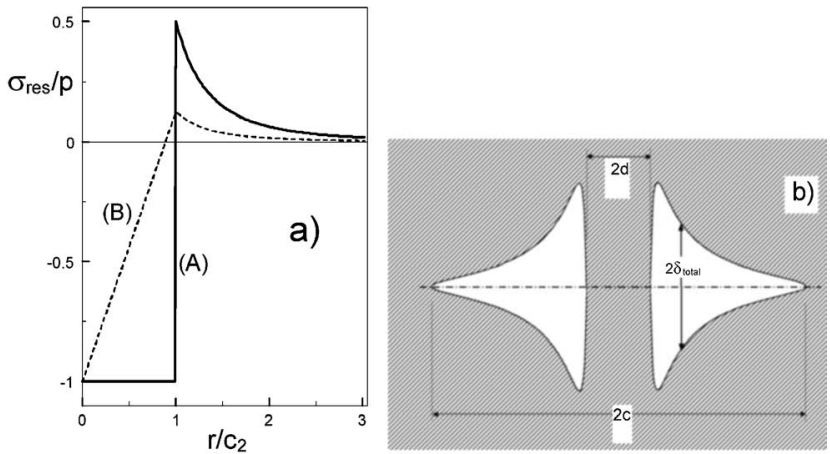

Fig. 2. (a) Residual stresses in the uncracked body for the limit cases $c_{1}=0$ (dashed curve) and $c_{1}=a$ (solid curve), (b) crack opening profile on the specimen surface (schematic representation).

where $r$ is the distance from the center of the half-sphere and $\sigma_{0}$ is the yield stress of the material. In the derivation of Eq. (1) the size parameter $c_{2}$ is related to the half-diagonal of the Vickers indentation $a$ that is assumed to be

$$
c_{2}=\alpha a, \alpha \approx 1
$$

and $c_{1}$ is the distance from the centre, within which the fully compressive stress state is reached. The parameters $c_{2}$ and $a$ are commonly chosen to be identical (i.e. $\alpha=1$ ). The equilibrium condition provides the coefficient $k$

$$
\int_{0}^{\infty} \sigma_{\text {res }}(r) r d r=0 \Rightarrow k=\frac{9 c_{2}^{2}}{c_{2}^{2}+c_{1} c_{2}+c_{1}^{2}}-1
$$

Now, two limit cases of Eq. (1) will be considered in more detail, namely,

$$
\begin{array}{ll}
\text { Case (A): } & c_{1}=c_{2} \\
\text { Case (B): } & c_{1}=0
\end{array}
$$

resulting in $k=2$ for (A) and $k=8$ for (B). The related stress distributions are plotted in Fig. $2(\mathrm{a})$ with the stresses normalised to the pressure $p$, defined by

$$
p=k \sigma_{0}
$$

The residual stresses $\sigma_{\text {res }}$ are the reason of the crack opening behaviour of Vickers indentation cracks, which is illustrated in Fig. 2(b). Limit case (A) was used in ${ }^{7)}$ for the computation of crack opening profiles. The inner boundary of the open crack, defining the contact radius $d$, can be measured from the COD profile observable on the specimen surface.

It is furthermore assumed that the crack can be represented as half of a fully embedded crack, neglecting free surface effects. This allows the application of the weight function for an internal crack. The residual stress intensity factor of a crack of radius $c$ is then given as ${ }^{8)}$

$$
K_{\text {res }}=\frac{2}{\sqrt{\pi c}} \int_{0}^{c} \frac{r \sigma_{\text {res }}(r) d r}{\sqrt{c^{2}-r^{2}}}<0
$$

and negative for any $c / a$. The crack-face displacement due to the residual stress field results from

$$
\delta_{\text {res }}(r)=\frac{4}{\pi E^{\prime}} \int_{r}^{c}\left(\int_{0}^{c^{\prime}} \frac{r^{\prime} \sigma_{\text {res }}\left(r^{\prime}\right)}{\sqrt{c^{\prime 2}-r^{\prime 2}}} d r^{\prime}\right) \frac{d c^{\prime}}{\sqrt{c^{\prime 2}-r^{2}}}<0
$$

with the Young's modulus for plane strain $E^{\prime}[7,8]$.

In a real structure, crack face penetration is not possible, of course. Prevented crack face penetration must result in a distribution of (positive) contact stresses $\sigma_{\text {cont }}$, causing a positive contact COD field $\delta_{\text {cont }}$

$$
\delta_{\text {cont }}(r)=\frac{4}{\pi E^{\prime}} \int_{r}^{c}\left(\int_{0}^{c^{\prime}} \frac{r^{\prime} \sigma_{\text {cont }}\left(r^{\prime}\right)}{\sqrt{c^{\prime 2}-r^{\prime 2}}} d r^{\prime}\right) \frac{d c^{\prime}}{\sqrt{c^{\prime 2}-r^{2}}}
$$

and a contact stress intensity factor

$$
K_{\text {cont }}=\frac{2}{\sqrt{\pi a}} \int_{0}^{c} \frac{r \sigma_{\text {cont }}(r) d r}{\sqrt{c^{2}-r^{2}}}
$$

This stress intensity factor is positive, since the contact stresses open the crack. The contact stresses must disappear in the region of real (positive) crack opening. In order to determine the contact stresses, the mixed boundary problem has to be solved

$$
\begin{array}{lll}
\delta_{\text {cont }}(r)+\delta_{\text {res }}(r)=0, \delta_{\text {cont }}>0 & \text { for } & r \leq d \\
\delta_{\text {cont }}(r)+\delta_{\text {res }}(r) \geq 0, \delta_{\text {cont }}=0 & \text { for } & r>d
\end{array}
$$

For the numerical treatment, a power series expansion (truncated after the term $N$ ) of the unknown contact stresses is applied according to

$$
\sigma_{\text {cont }}=\left\{\begin{array}{cc}
\sum_{n=1}^{N} A_{n}\left[1-(r / d)^{2 n}\right] & \text { for } r \leq d \\
0 & \text { for } r>d
\end{array}\right.
$$

with an estimation of $d$. The $N$ unknown coefficients $A_{n}$ for each contact radius $d$ can be determined exclusively from the condition $\delta_{\text {total }}=0$ for $r<d$. The displacements were evaluated at $m>N$ points in $0<r<d$. From the minimised expression

$$
\sum_{n=1}^{N}\left(\delta_{\text {res }}(r)+\delta_{\text {cont }}(r)\right)^{2}=\min
$$

the coefficients $A_{n}$ were determined. That contact radius, for which the smallest square sum is obtained, is the solution for d.

An approximate representation of $\delta_{\text {cont }}$ by a single term of the type (12) was given in ${ }^{7)}$ for the limit case (A) and $c / c_{2}>2.5$ as

$$
\sigma_{\text {cont }} \cong D\left(1-\left(r / c_{2}\right)^{2 q}\right)
$$

with

$$
D \cong p\left(0.89+0.11 c_{2} / c\right)
$$

and

$$
q \cong 5.32+2.69 c_{2} / c+13.33\left(c_{2} / c\right)^{2} .
$$

From the present computations, it can be concluded that the dependencies (14b) and (14c) can be extended to $c / c_{2} \geq 1.5$. The contact stresses for case (B) have a more complicated behaviour and are not given here as a fit function.

\section{Total stress intensity factor}

The total stresses $\sigma_{\text {total }}$ result from the superposition of the residual and the contact stresses

$$
\sigma_{\text {total }}=\sigma_{\text {res }}+\sigma_{\text {cont }}
$$

The really active total stress intensity factor $K_{\text {total }}$ is then

$$
K_{\text {total }}=K_{\text {res }}+K_{\text {cont }}
$$

The small symbols in Fig. 3 (a) represent the stress intensity factors computed for the two limit cases. An approximate fit of the numerical data for case (A) may be expressed by

$$
K_{(\mathrm{A})} \cong K_{\max ,(\mathrm{A})} \tan h\left[\sqrt{3\left(\frac{c}{c_{2}}-1\right)}\right]
$$

as represented by the dashed curve in Fig. 3(a). For case (B) a representation by 


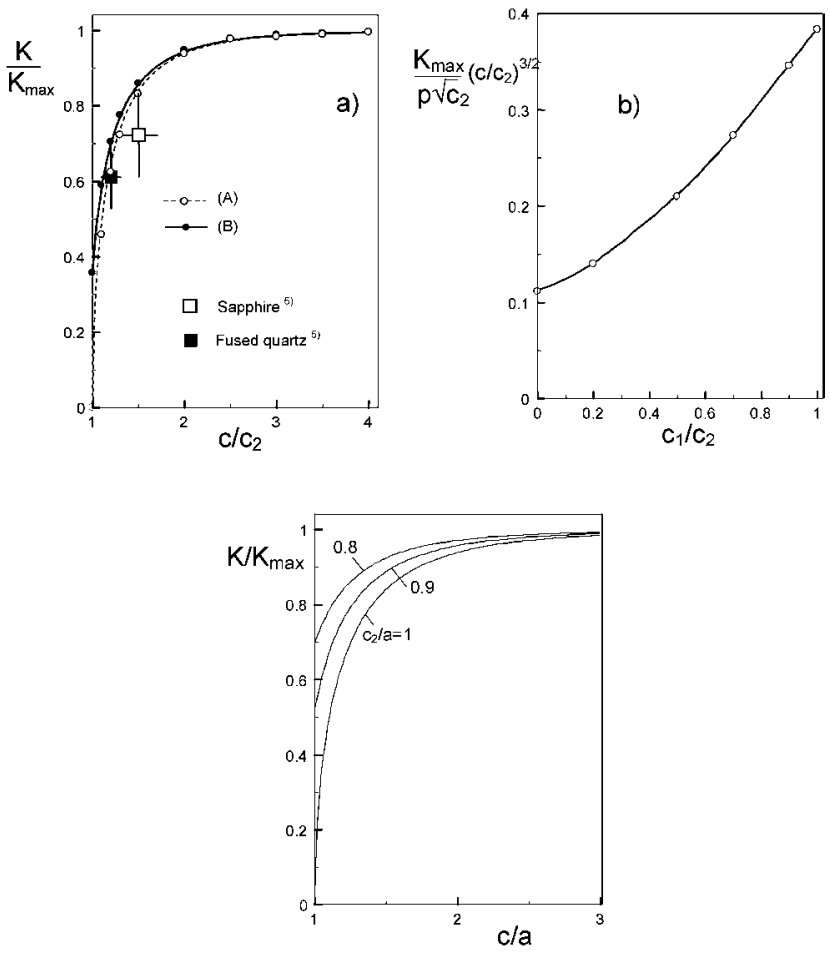

Fig. 3. (a) Normalised total stress intensity factor (open symbols: contact zone for case (A) $\left(c_{1}=c_{2}\right)$, full symbols: $K$ for case (B) $\left(c_{1}=\right.$ 0 ), (b) asymptotic stress intensity factor vs. $c_{1} / c_{2}$, (c) influence of the ratio $\alpha=c_{2} / a$ on the stress intensity factor.

$$
K_{(\mathrm{B})} \cong K_{\max ,(\mathrm{B})} \tan h\left[\sqrt{3\left(\frac{c}{c_{2}}-1+0.05\right)}\right]
$$

is proposed, shown as the solid curve of Fig. 3 (a). In both cases the reasoning for the analytical approximation is the following. For small $c / c_{2}$ values a square-root behaviour is fracture mechanically reasonable and an asymptotic approach to $K_{\max }$ should happen for long $c / c_{2}$-values. As a consequence a tanh-function fitted to the numerical values leads to errors in $K_{(\mathrm{A})} / K_{\max ,(\mathrm{A})}$ of less than 0.02 for any $c / c_{2}$.

From these results, it becomes obvious that the influence of the a priori unknown parameter $c_{1} / a$ on the stress intensity factor is negligible. The maximum values $K_{\max }$ are plotted in Fig. 3(b) versus the parameter $c_{1}$. A fit relation for this dependence is

$$
\begin{aligned}
& K_{\max } \frac{\left(c / c_{2}\right)^{3 / 2}}{p \sqrt{c_{2}}}=0.111 \\
& +0.0973\left(\frac{c_{1}}{c_{2}}\right)+0.2293\left(\frac{c_{1}}{c_{2}}\right)^{2}-0.0540\left(\frac{c_{1}}{c_{2}}\right)^{3}
\end{aligned}
$$

The variation of the fracture mechanics weight function for different crack types (e.g. cruciform cracks compared to single semi-circular cracks) of course affects the value of the stress intensity factor. Nevertheless, the ratio $K / K_{\max }$ is much less sensitive to the special weight function since the numerator and the denominator of this ratio are influenced in the same way.

The experimental results from, ${ }^{5)}$ together with the expected error bars, are introduced in Fig. 3(a) as the squares. Results obtained in ${ }^{5)}$ for barium titanate are ignored here, because piezoelectric materials have a strong non-linear stress-strain behaviour and a significant change of the Young's modulus
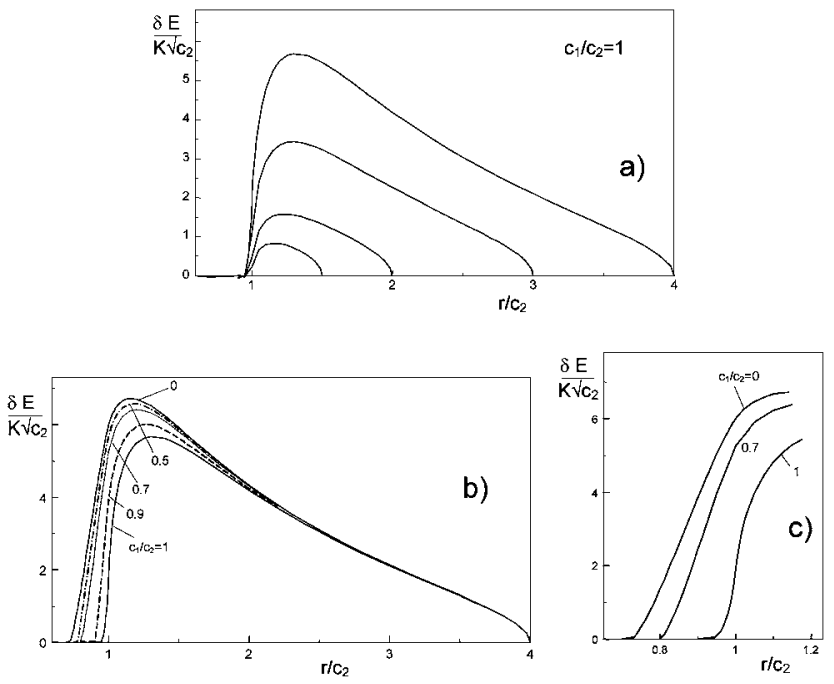

Fig. 4. Crack opening displacement of Vickers indentation cracks, (a) influence of the crack size, (b) influence of the distribution of residual stresses, characterised by the parameter $c_{1}$ in Eq. (3), (c) details of CODs near the contact zones.

during the loading/unloading cycle. Therefore, such a material does not allow for a simple elastic/plastic analysis. For fused quartz at least a good agreement between experiment and computations can be observed. Even though the error bar of the experimetal values of sapphire just touches the theoretically predicted curve the general trend of the experimental and theoretical results are identical.

For the computed curves in Fig. $3(\mathrm{a})$, it was assumed $c_{2}=a$, i.e. $\alpha=1$. In order to show the influence of $c_{2} \neq a$, the curves of Fig. 3(c) were computed for different values of $\alpha=c_{2} / a$. The effect of $\alpha<1$ is an onset of the curve $K / K_{\max }=f(c / a)$ at a finite value $K / K_{\max }>0$ with a more moderate further increase of the curve. The maximum value $K_{\max }$ for $c_{1}=c_{2}$ is

$$
K_{\max ,(\mathrm{A})}(\alpha)=\alpha^{2} K_{\max ,(\mathrm{A})}(\alpha=1)
$$

\section{Crack opening displacement}

With the set of coefficients $A_{n}$ of Eq. (12), the total displacements $\delta_{\text {total }}=\delta_{\text {cont }}+\delta_{\text {res }}$ were computed also outside the contact zone. Figure $4(\mathrm{a})$ shows the COD fields for the limit case (A) at variable relative crack lengths $c / c_{2}$. In Fig. 4(b) the influence of the free parameter $c_{1}$ is illustrated. For any choice of this parameter, the same COD field for $r / c_{2}>1.8$ and $c / c_{2}>3$ in the "far field" is obtained. This fact is a direct consequence of the Saint Venant theorem and was the reason why the authors in $^{7)}$ restricted their studies to the limit case (A). Figure 4(c) shows the COD close to the contact zones.

An approximate analytical description of the crack opening displacement field is given in $^{7)}$ as

$$
\delta \cong \frac{4 c}{\pi E^{\prime}}\left[\left(p^{*}-p\right) g_{1}\left(r, c, c_{2}\right)+\frac{c_{2}}{2 c} g_{2}\left(r, c, c_{2}\right)\right]
$$

with the functions

$$
\begin{gathered}
g_{1}\left(c, c_{2}, r\right)=\sqrt{1-\left(\frac{r}{c}\right)^{2}}\left(1-\sqrt{1-\left(\frac{c_{2}}{c}\right)^{2}}\right)+ \\
+\frac{r}{c}\left[E\left(\left(\frac{c_{2}}{r}\right)^{2}\right)-E\left(\arcsin \frac{r}{c},\left(\frac{c_{2}}{r}\right)^{2}\right)\right.
\end{gathered}
$$



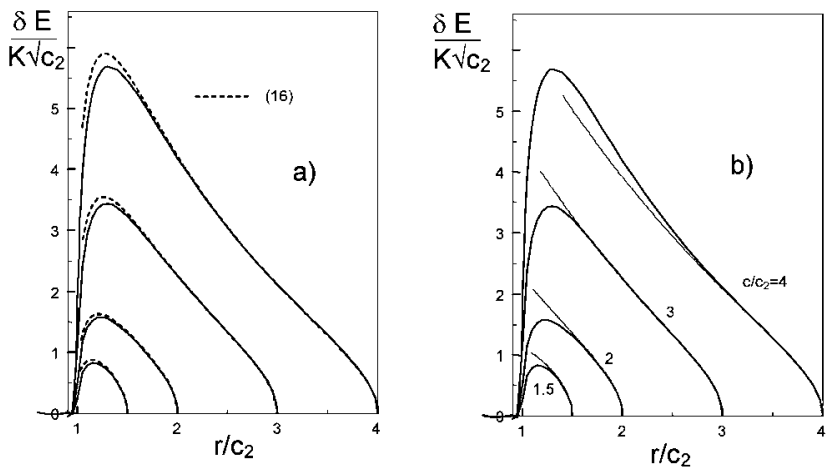

Fig. 5. Total COD: a) comparison of the numerical solution (solid curve) with the approximation Eq. (20a) - (20e) (dashed curves), (b) approximation by a truncated power series (21) including the coefficients $A_{0}-A_{2}$.

$$
\begin{aligned}
& -\left(1-\left(\frac{c_{2}}{r}\right)^{2}\right)\left(K\left(\left(\frac{c_{2}}{r}\right)^{2}\right)\right. \\
& \left.\left.-F\left(\arcsin \frac{r}{c},\left(\frac{c_{2}}{r}\right)^{2}\right)\right)\right] \\
& g_{2}\left(c, c_{2}, r\right)=\frac{c_{2}}{r}\left[E\left(\left(\frac{c_{2}}{r}\right)^{2}\right)-E\left(\arcsin \frac{r}{c},\left(\frac{c_{2}}{r}\right)^{2}\right)\right]
\end{aligned}
$$

In (20a) $p^{*}$ is that constant pressure in $0<r<c_{2}$ that yields the same stress intensity factor as the more complicated contact stress distribution. It is given by

$$
\begin{aligned}
\frac{p^{*}}{p}= & \frac{2-\sqrt{1-\left(\frac{c_{2}}{c}\right)^{2}}\left(2+\left(\frac{c_{2}}{c}\right)^{2}\right)}{2\left(1-\sqrt{\left.1-\left(c_{2} / c\right)^{2}\right)}\right.} \\
& +\frac{0.383\left(\frac{c_{2}}{c}\right)^{2} \sqrt{\pi} \tanh \left(\sqrt{\left.3\left(c / c_{2}-1\right)\right)}\right.}{2\left(1-\sqrt{\left.1-\left(c_{2} / c\right)^{2}\right)}\right.}
\end{aligned}
$$

and $\frac{p^{*}}{p} \cong 0.6602+0.1643 c_{2} / c+0.1755\left(c_{2} / c\right)^{2}$

Figure 5(a) shows the approximate displacement field according to Eqs. (20a)-(20c) as the dashed curves in comparison with the numerical solution as the continuous curves. Differences occur very close to the contact zone only, i.e. for $r \approx c_{2}$.

In ${ }^{7)}$ a series expansion for the displacements was proposed. In order to include results for cracks with $c / c_{2}<2.5$, this expansion has been modified as

$$
\begin{aligned}
\frac{\delta}{K}= & \frac{\sqrt{c_{2}}}{E^{\prime}}\left(A_{0} \sqrt{\frac{x}{c_{2}}}+A_{1}\left(\frac{x}{c_{2}}\right)^{3 / 2}+A_{2}\left(\frac{x}{c_{2}}\right)^{5 / 2}\right. \\
& \left.+\cdots+A_{n}\left(\frac{x}{c_{2}}\right)^{n+1 / 2}\right)
\end{aligned}
$$

with

$$
A_{0}=\sqrt{\frac{8}{\pi}}
$$

The first higher-order coefficients are compiled in Table 1. Fit relations for $A_{1}$ and $A_{2}$ are
Table 1. Coefficients for the Power Series Expansion of the COD Field According to Eq. (21)

\begin{tabular}{|l|l|l|l|}
\hline $\mathrm{c} / \mathrm{c}_{2}$ & $\mathrm{~A}_{1}$ & $\mathrm{~A}_{2}$ & $\mathrm{~A}_{3}$ \\
\hline 1.5 & 0.3377 & -0.73638 & \\
2.5 & 0.5941 & 0.08872 & \\
3 & 0.5638 & 0.15189 & 0.01775 \\
3.5 & 0.5050 & 0.13585 & 0.02945 \\
4 & 0.4499 & 0.11222 & 0.02511 \\
5 & 0.4030 & 0.09182 & 0.01951 \\
6 & 0.3310 & 0.06305 & 0.01154 \\
8 & 0.2796 & 0.04536 & 0.00715 \\
& 0.2125 & 0.02638 & 0.00321 \\
\hline
\end{tabular}

$$
A_{1} \cong 11.7 \exp \left[-2.063\left(c / c_{2}-1\right)^{0.28}\right]-\frac{0.898}{c / c_{2}-1},
$$

$$
A_{2} \cong 44.5 \exp \left[-3.712\left(c / c_{2}-1\right)^{0.28}\right]-\frac{1}{\left(c / c_{2}-1\right)^{3 / 2}}
$$

with a validity range of $c / c_{2} \geq 1.4$. Figure 5(b) shows the approximation (21) including the coefficients $A_{0}-A_{2}$ (dashed curves), together with the numerical results (solid curves). This degree of approximation is sufficient for cracks with $c / c_{2}$ $\leq 3$. For $c / c_{2}>3$, the additional coefficient $A_{3}$ of Table 1 may be included. The convergence of approximation is illustrated in Fig. 6 for a crack with $c / c_{2}=4$. Use of $A_{0}$ exclusively gives the so-called Irwin parabola (dashed curve). Use of $A_{0}$ and $A_{1}$ yields the dash-dotted curve and $A_{0}-A_{2}$ the thin continuous curve. Finally, the dotted curve represents the solution including $A_{0}-A_{3}$.

A good approximation including the coefficients $A_{n}$ up to $A_{2}$ is obtained for $c-r<\left(c-c_{2}\right) / 3$ at least. In the case of short cracks with $c / c_{2}<3.5$, the accuracy is sufficient even in the range of $c-r<2\left(c-c^{2}\right) / 3$.

\section{Estimation of the residual stress parameter $p$}

The pressure- $p$ in the centre region of the Vickers impression can be estimated by comparing the measurements with the results of the computations. From (17a) and (18), it results for the limit case $(\mathrm{A})$ by introducing $c_{1} / c_{2}=1$ and identifying $c_{2}$ with the pyramid half-diagonal $a$ :

$$
\frac{K}{p \sqrt{a}(a / c)^{3 / 2}} \cong 0.383
$$

Comparing this with the well-established empirical relation using the factor of proportionality by Anstis et al. ${ }^{2}$ 


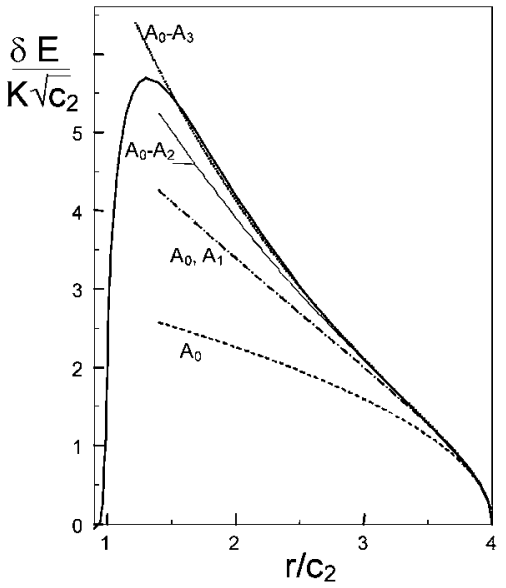

Fig. 6. Total COD: convergence behaviour of (21). Solutions including an increasing number of coefficients $A_{\mathrm{n}}$.

$$
K=0.016 \frac{P}{c^{3 / 2}} \sqrt{\frac{E}{H}}
$$

yields by use of the hardness $H$

$$
H=\frac{P}{2 a^{2}}
$$

and with the rough estimation of $H \cong E / 10$ as found for the materials investigated in ${ }^{5)}$ :

$$
\Rightarrow p=0.083 \sqrt{H E} \cong 0.26 H
$$

This result can be compared with relation of the Meyers hardness to the yield stress of an ideal plastic material for a flat punch situation applying the Huber-Mises criterion, $H \approx$ $\left.3 \sigma_{0} \cdot{ }^{9}\right)$ Because we have with Eq. (6) $p=2 \sigma_{0}$ the result of Eq. (28) is equivalent to $H \approx 8 \sigma_{0}$, which is the right order of magnitude.

\section{Conclusions}

The stress intensity factor relation for pyramidal indentations was determined for cracks with small values of the ratio $c / a$. In addition, the related crack opening displacement field was computed. The main results of our analysis are:

(1) The empirical proportionality coefficient in the $K \propto$ $P / c^{3 / 2}$ relation that is constant for cracks with $c / a>3$ decreases with $c / a \rightarrow 1$. The quantitative value of course is dependent on the chosen pressure distribution directly below the indenter, but the general trend confirms well the experimental findings in. ${ }^{5)}$

(2) It was also demonstrated that the COD field for $c / a$ $<3$ can be described by the same relations as those given in ${ }^{7)}$ for $c / a>3$.

(3) A series expansion of COD similar to ${ }^{7)}$ is proposed. The coefficients were modified to allow for an application to $c / a<3$.

\section{References}

1) Lawn, B. R., Evans, A. G. and Marshall, D. B., J. Am. Ceram. Soc., Vol. 63, pp. 574-581 (1980).

2) Anstis, G. R., Chantikul, P., Lawn, B. R. and Marshall, D. B., J. Am. Ceram. Soc., Vol. 64, pp. 533-538 (1981).

3) Harding, D. S., Oliver, W. C. and Pharr, G. M., Mater. Res. Soc. Symp. Proc., Vol. 356, pp. 663-668 (1995).

4) Niihara, K., Morena, R. and Hasselman, D. P. H., J. Mater. Sci. Letters, Vol. 1, pp. 13-16 (1982).

5) Scholz, T., Schneider, G. A., Munoz-Saldana, J. and Swain, M. V., Appl. Phys. Letters, Vol. 84, pp. 3055-3057 (2004).

6) Hill, R., "Mathematical Theory of Plasticity," Oxford University Press, Oxford (1950).

7) Fett, T., Kounga Njiwa, A. B. and Rödel, J., Engng. Fract. Mech., Vol. 72, pp. 647-659 (2005).

8) Sneddon, I. N., Proc. of the Royal Soc. London Ser. A, Vol. 187, pp. 229-229 (1946).

9) Tabor, D., "The Hardness of Metals," Oxford University Press, Oxford (1951). 